DOI: https://doi.org/10.47405/aswj.v6i5.183

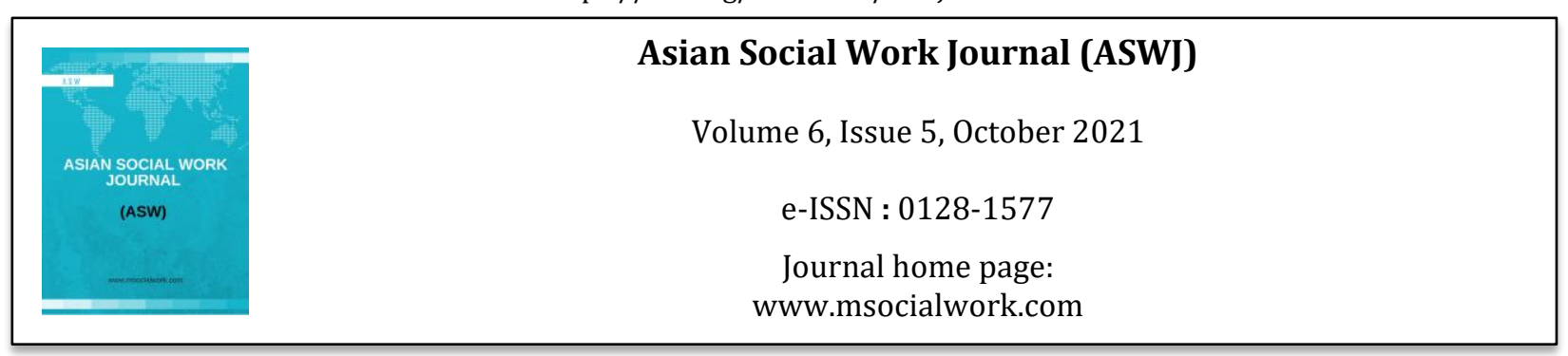

\title{
Person Centered and Trauma Informed Approach in Social Work Practice and Ways to Ensure Diversity in both Approaches
}

\author{
Niket Paudel ${ }^{1}$ \\ 1Independent Scholar \\ Correspondence: Niket Paudel (niket.paudel@gmail.com)
}

\begin{abstract}
Person-Centered approach is an adapted work of Carl Rogers, where the focus was mainly on psychotherapy and counselling. Rogers believed, the basic nature of an individual is constructive and trustworthy - given that the individual is freely functioning. Person-Centered approach is the backbone of social work practice alongside trauma-informed approach. Any emotionally and physically daunting events that affects the response of an individual is trauma. Trauma damages the freely functioning state of an individual - emotionally, socially and behaviorally. By introducing an approach that is traumainformed will help social worker to not only understand the emotions of the individual while working with them but also guide the social work practice in better understanding while working with the individuals.
\end{abstract}

Keywords: person-centered approach, trauma informed approach, trauma, congruence, encouragers, unconditional positive regard

\section{Person Centered Approach}

Cultivated from the notions of a humanistic approach, person centered approach views people as adept and competent to resolve their impediments and challenges, recognize their potential, and shift lives in positive ways (Tudor, Keemar, Tudor, Valentine, \& Worrall, 2004). Carl Rogers, the major contributor of Person-Centered Approach not only emphasized the humanistic perspectives but also added therapeutic relationships with clients to promote self-esteem and help them use their strengths (Mearns, Thorne, \& McLeod, 2013). In person-centered approach, those who come for the therapy are not referred to as patients but as clients as both the social worker and the clients are viewed as equal partners in the therapeutic process (Murphy, Duggan, \& Joseph, 2013). Furthermore, the client is responsible for improving their lives and not the social worker, the decisions will be made by the client for themselves (Murphy, Duggan, \& Joseph, 2013). The power dynamics between the social worker and the client are balanced in this approach (Hagner, May, Kurtz, \& Cloutier, 2014). Also, no particular techniques can be used in this approach as every client is unique as they have their own individuality (Hagner, May, Kurtz, \& Cloutier, 2014).

Some of the key Person Centered skills a social worker needs to consider when engaging with clients in the mental health and disability context are congruence, unconditional positive regard, acceptance, empathy, and non-directiveness (Seligman \& Reichenberg, 2006).

Congruence, in a person-centered approach, is the authenticity and genuineness of the social worker towards the client (Joseph \& Murphy, 2013). As a social worker, not only the direct form of 
communication but also body language and non-verbal communication is vital when working with clients (Sakroni, 2016). For example, a person with mental health issues shares their struggles, and the social worker fidgets their hands and looks somewhere else instead of paying attention, the client might not be able to build a relationship with the social worker as they might think they are not being listened or the social worker might not seem genuine to the client. Skills like using open-ended questions and paraphrasing what the client says might be helpful as that would suggest, the social worker is attentive and is listening (Australian Institute of Professional Counsellors, 2010). Paraphrasing also makes the client feel they are being listened to and the social worker is willing to help. Another vital skill needed when working in the mental health field is the use of encouragers (Australian Institute of Professional Counsellors, 2010). Encouragers like "hmm", "what else" might help the client to open up more, especially if the client is struggling to open up (Australian Institute of Professional Counsellors, 2010). Encouragers work like magic when working with shy and introverted clients alongside people with mental health issues (Kabir, 2017). People with mental health issues and disabilities are vulnerable; encouragers might help them share their emotions accurately (Kabir, 2017).

Unconditional positive regard indicates to the social worker accepting and respecting their clients which does not necessarily mean the social worker has to agree with everything the client says but acknowledging the client and whatever the client is doing and saying, rather than disagreeing with them (Australian Institute of Professional Counsellors, 2010). Unconditional positive regard helps the client to open up and facilitate the intervention as the client would feel they are not being judged and being accepted for who they are (Australian Institute of Professional Counsellors, 2010). Non-directiveness is another skill that is important in a person-centered approach (Australian Institute of Professional Counsellors, 2010). Non-directiveness refers to the client being the focus in the process rather than the social worker giving strategies (Australian Institute of Professional Counsellors, 2010). For example, a person with a disability being asked what are the things that they want to work on instead of laying the intervention by the social worker and telling them to follow it. Empathizing with the client and accepting who they are is another major skill required when working in mental health and disability (Australian Institute of Professional Counsellors, 2010). In the healing process, the client should have a welcoming environment to express their emotions (Australian Institute of Professional Counsellors, 2010). To express their emotions, the social worker should accept their emotions, both positive and negative. As the client might be wrestling to deal with the situation, the social worker should be aware of the emotions and accepting them (Australian Institute of Professional Counsellors, 2010). For example, a client is struggling in maintaining a healthy relationship with their partner due to their mental health issues where the client has mental breakdown often; the social worker should be mindful about the impact of the mental health issues having on the client which might be affecting the relationship.

\section{Trauma Informed Approach}

Trauma Informed Approach is not just a concept or a theory, rather it aims to shift the thinking of how an individual is viewed by not only understanding the human behaviour and their coping skills but also examining their traumatic events throughout their life from past to present (Substance Abuse and Mental Health Services Administration, 2014). The approach aims at understanding the individual's functioning from past to present and investigates why the problems exist rather than just seeing the presenting problems (Knight, 2015). The approach is strengths-based approach (Kimberg \& Wheeler, 2019). By doing a systematic screening of the life events of an individual without doing any harm to the client, the approach works to avoid any foreseeable future traumatic events by working on the strengths of the client (Kimberg \& Wheeler, 2019). Trauma informed approach is different from trauma-centered intervention as the latter focuses mainly on the underlying trauma whereas the former assists the individual by "developing their capacities for managing distress and for engaging in more effective daily functioning', (Gold, 2001).

Some of the skills of trauma-informed approach in mental health and disability are active listening, acknowledging and validating, and non-judgmental (Sweeney, Filson, Kennedy, Collinson, \& Gillard, 2018). 


\section{Asian Social Work Journal (ASWJ), Volume 6, Issue 5, (page 21 - 24), 2021 \\ DOI: https://doi.org/10.47405/aswj.v6i5.183}

Active Listening: Active listening is a way of communicating and responding to an individual that promotes mutual communication. Active listening in simple terms can be defined as not interrupting while the other individual is sharing their experiences (University of Adelaide, 2014). It allows the social worker to connect with the client as the client feels listened to (Karpetis, 2017). The client feels validated when the social worker just listens to their traumatic past without interrupting them (Ferencik \& Ramirez-Hammond, 2013). It is not uncommon to share own experiences while listening to the other individual to show the common ground, but while doing so will shift the focus on the social worker and not the client which will prevent the client to share more of their experiences. Listening to the clients actively helps in giving the power to the client rather than keeping it with the social worker (Ferencik \& Ramirez-Hammond, 2013).

Acknowledging and Validating: Lack of active listening might lead to a difficult experience for the client (Ferencik \& Ramirez-Hammond, 2013). This might lead to downplaying the client's trauma. Every individual possesses a distinct individualistic character and has their own story (Ferencik \& Ramirez-Hammond, 2013). It is vital to acknowledge the client's experience (Purkey, Patel, \& Phillips, 2018). Furthermore, trauma might vary from person to person. Research has suggested that "a person's feelings about their traumatic experiences in childhood are just sympathetically listened to and validated, and their pain and suffering as a result of their trauma is acknowledged and authenticated, their condition improves" (Hosier, 2016). Other skills and interventions might help an individual but it cannot be done without acknowledging and validating the client's experience (Purkey, Patel, \& Phillips, 2018). Doing so also provides a safe space for the client to share their life story and speak about their trauma (Purkey, Patel, \& Phillips, 2018).

Non-judgemental: Similarly, being non-judgmental is another key skill when working in a traumainformed space (Briere \& Scott, 2014). A client comes to see a social worker to have a safe space and the social worker must understand that (Briere \& Scott, 2014). Social workers should be aware of their bias and avoid being judgemental (Australian Association of Social Workers, 2020). For example, social workers must be aware of the impact of trauma on the male clients by understanding there are great gender differences when it comes to experiences of men and their response flight with trauma (Covington, Griffin, \& Dauer, 2011).

\section{Ensuring Diversity in both approaches}

Most of the studies suggest that any counselling approach is a Western approach and can only be applicable in the culture that holds the idea of an independent self (Kim, 2018). On the other hand, some studies have suggested such a view as binary and ethnic stereotyping (Kim, 2018). To back it up, some Japanese researchers have even stated that "Japanese are just as independent as Americans, or in some cases, more independent" (Markus \& Kitayama, 2003, p. 282). Though people are influenced by culture, race, gender, religion, but at an individual level, everybody possesses certain differences and uniqueness (Kim, 2018). Hence, social workers should be aware of people's individuality alongside, their culture and world values (Sue, Sue, Neville, \& Smith, 2019). Being aware of people's individuality does not mean disregarding cultural aspects rather it suggests using a holistic approach to understand client's issues which is only possible by respecting both their uniqueness and cultural influences (Kim, 2018). Cultural competence is a major factor in ensuring diversity which involves (Casemore, 2011):

i. Social workers being aware of their biases and cultural values,

ii. Cross-cultural skills and

iii. Knowledge of client's cultural values

\section{References}

Australian Association of Social Workers. (2020). Code of Ethics. Melbourne: Australian Association of Social Workers.

Australian Institute of Professional Counsellors. (2010). Person-centred Therapy: A GUIDE TO COUNSELLING THERAPIES (DVD). Fortitude Valley: J \& S Garrett Pty Ltd . 
DOI: https://doi.org/10.47405/aswj.v6i5.183

Briere, J., \& Scott, C. (2014). Principles of Trauma Therapy: A Guide to Symptoms, Evaluation, and Treatment (4th ed.). Thousand Oaks: Sage Publications.

Casemore, R. (2011). Person-Centred Counselling in a Nutshell (2 ed.). New York: SAGE Publications Ltd.

Covington, S., Griffin, D., \& Dauer, R. (2011). Helping Men Recover: A Program for Treating Addiction (1st ed.). Hoboken: John Wiley \& Sons Inc.

Ferencik, S. D., \& Ramirez-Hammond, R. (2013). Trauma Informed Care: Best Practices and Protocols for Ohio's Domestic Violence Programs. Columbus: Ohio Domestic Violence Network (ODVN).

Gold, S. N. (2001). Conceptualizing child sexual abuse in interpersonal context: Recovery of people, not memories. Journal of Child Sexual Abuse, 10(1), 51-71.

Hagner, D., May, J., Kurtz, A., \& Cloutier, H. (2014). Person-Centered Planning for Transition-Aged Youth with Autism Spectrum Disorders. Journal ofRehabilitation, 80(1), 4-10.

Hosier, D. (2016). The vital importance of having our traumatic experiences validated. Childhood Trauma Recovery. Retrieved 2021

Joseph, S., \& Murphy, D. (2013). Person-Centered Approach, Positive Psychology, and Relational Helping: Building Bridges. Journal of Humanistic Psychology, 53(1), 26-51.

Kabir, S. M. (2017). Communication Skills. In Essentials of Counseling (pp. 95-116). Dhaka: Abosar Prokashana Sangstha.

Karpetis, G. (2017). Social Work Skills: A Narrative Review of the Literature. British Journal of Social Work, 48(3), 1-20.

Kim, J. (2018). Consideration of the applicability of personcentered therapy to culturally varying clients, focusing on the actualizing tendency and selfactualization - from East Asian perspective. Person-Centered \& Experiential Psychotherapies, 17(3), 201-223.

Kimberg, L., \& Wheeler, M. (2019). Trauma and Trauma-Informed Care. In Trauma-Informed Healthcare Approaches (pp. 25-56). Cham: Springer.

Knight, C. (2015). Trauma-Informed Social Work Practice: Practice Considerations and Challenges. Clinical Social Work Journal, 43(1), 25-37.

Markus, H. R., \& Kitayama, S. (2003). Culture, self and the reality of the social. Psychological Inquiry, 14(3 and 4), 277-283.

Mearns, D., Thorne, B., \& McLeod, J. (2013). Person-Centred Counselling in Action (4th ed.). Thousand Oaks: SAGE Publications Ltd.

Murphy, D., Duggan, M., \& Joseph, S. (2013). Relationship-Based Social Work and Its Compatibility with the Person-Centred Approach: Principled versus Instrumental Perspectives. The British Journal of Social Work, 43(4), 703-719.

Purkey, E., Patel, R., \& Phillips, S. P. (2018). Trauma-informed care: Better care for everyone. Canadian Family Physician, 64(3), 170-172.

Sakroni, S. (2016). The Effort Of Social Workers To Nurture Verbal And Non-Verbal Communication On Client Participation Level. The International Journal of Social Sciences and Humanities Invention, 3(3), 1930-1937.

Seligman, L. W., \& Reichenberg, L. W. (2006). Theories of counseling and psychotherapy: Systems, strategies, and skills (4th Edition ed.). New Jersey: Pearson Education.

Substance Abuse and Mental Health Services Administration. (2014). Trauma-Informed Care in Behavioral Health Services. Rockville: SAMHSA.

Sue, D. W., Sue, D., Neville, H. A., \& Smith, L. (2019). Counseling the Culturally Diverse: Theory and Practice (8 ed.). John Wiley \& Sons Inc: Hoboken.

Sweeney, A., Filson, B., Kennedy, A., Collinson, L., \& Gillard, S. (2018). A paradigm shift: relationships in trauma-informed mental health services. BJPsych advances, 24(5), 319-333.

Tudor, L. E., Keemar, K., Tudor, K., Valentine, J., \& Worrall, M. (2004). The Person-Centred Approach: A Contemporary Introduction (1 ed.). Bedford: Red Globe Press.

University of Adelaide. (2014). Active Listening. Adelaide: University of Adelaide. 